This manuscript has been accepted for publication in Psychoneuroendocrinology. It has not undergone copy-editing. (C) 2021. This manuscript version is made available under the CC-BY-NCND 4.0 license http://creativecommons.org/licenses/by-nc-nd/4.0/

\title{
Genetic variation in the oxytocin system and its link to social motivation in human infants
}

Kathleen M. Krol ${ }^{1,2 * \dagger}$, Nauder Namaky ${ }^{1 \dagger}$, Mikhail V. Monakhov ${ }^{3,4}$, Poh San Lai ${ }^{5}$, Richard Ebstein ${ }^{4,6}$, and Tobias Grossmann ${ }^{1,2}$

${ }^{1}$ Department of Psychology, University of Virginia, USA; ${ }^{2}$ Max Planck Institute for Human Cognitive and Brain Sciences, Germany; ${ }^{3}$ Department of Neuroscience, Baylor College of Medicine, USA; ${ }^{4}$ Department of Psychology, National University of Singapore, Singapore; ${ }^{5}$ Department of Pediatrics, National University of Singapore, Singapore; ${ }^{6}$ China Center for Behavior Economics and Finance, Southwestern University of Finance and Economics, China

*To whom correspondence can be addressed:

Kathleen M. Krol

485 McCormick Rd., Charlottesville, VA 22904 USA

Phone: +1 (434) 982-4439

Email: $\underline{\text { krol@ virginia.edu }}$

†These authors contributed equally.

Short title: $C D 38$ genotype impacts infant motivational indices 


\begin{abstract}
Frontal brain asymmetry has been linked to motivational processes in infants and adults, with left lateralization reflecting motivation to approach and right lateralization reflecting motivation to withdraw. We examined the hypothesis that variability in infants' social motivation may be linked to genetic variation in the oxytocin system. Eleven-month-old infants' brain responses and looking preferences to smiling and frowning individuals were assessed in conjunction with a polymorphism in $C D 38$ (rs3796863) linked to autism spectrum disorder (ASD) and reduced oxytocin. Frontal brain asymmetry and looking preferences differed as a function of $C D 38$ genotype. While non-risk A-allele carriers displayed left lateralization to smiling faces (approach) and a heightened looking preference for the individual who smiled, infants with the CC (ASD risk) genotype displayed withdrawal from smiling faces and a preference for the individual who frowned. Findings demonstrate that the oxytocin system is linked to brain and behavioral markers of social motivation in infancy.
\end{abstract}

Keywords: frontal asymmetry; oxytocin; fNIRS; eyetracking; infant; emotion 


\section{Introduction}

Infancy is a developmental period that may be of critical importance in the etiology of psychiatric disorders characterized by reduced social motivation and withdrawal, such as autism spectrum disorder (ASD), major depressive disorder (MDD), and social anxiety disorder (SAD). Social evaluation and impression formation are foundational socio-cognitive skills enabling the effective detection and responding to potential interaction partners. Behavioral studies have shown that by 6 months of age infants evaluate others and form impressions that influence their social motivation, as displayed in a preference for helpful and an avoidance of hindering agents (Hamlin et al., 2007). Around 7 months of age, infants detect eye contact and can discriminate between smiles and frowns (Grossmann, 2013, 2017; Krol et al., 2015a), and by at least 11 months of age, infants are capable of using these facial cues to form social impressions that guide their behavioral preferences (Krol and Grossmann, 2020). However, already in infancy variability may exist with respect to such foundational social-motivational tendencies. For example, recent work has demonstrated that infants vary in their preference for prosocial agents when compared to antisocial agents (Cowell and Decety, 2015; Krol and Grossmann, 2020). In fact, considering that reduced social approach or increased social withdrawal in 6-month-old infants has been shown to be associated with diminished cognitive, language, and social skills at 30 months (Milne et al., 2009), it is possible that early-developing differences in social motivation may foreshadow long-term deficits in social development (Chevallier et al., 2012). Thus, identifying and elucidating factors that might contribute to infants' motivation to approach or withdraw from others is of critical importance in informing our understanding of typical and atypical social development. The current study is the first to examine potential neural, behavioral, and genetic factors involved in the development of disorders characterized by social withdrawal during this sensitive period of human development.

Frontal brain asymmetry has been linked to motivational processes in adults and infants, with greater left frontal asymmetry reflecting motivation to approach and greater right frontal asymmetry reflecting motivation to withdraw (for a review, see Harmon-Jones and Gable, 2018). In neurotypical adults, shifts in frontal brain lateralization, indexed by EEG frontal alpha asymmetry (FAA), have been 
linked to motivational approach and avoidance tendencies when producing facial expressions of emotion (Coan et al., 2001), and in infants as young as 10 months, greater left FAA - indexing approach - is elicited upon perception of smiling facial expressions (Davidson and Fox, 1982). Greater right FAA, both at rest and during emotional challenge, has been associated with increased risk of psychopathology characterized by social withdrawal. For example, greater right FAA has been reported in adults diagnosed with MDD (Coan and Allen, 2003; Grunewald et al., 2018; Stewart et al., 2010; Stewart et al., 2011, 2014; Thibodeau et al., 2006) and SAD (Davidson et al., 2000). Recent work in children diagnosed with ASD indicates greater right FAA in response to direct-gaze faces and greater left FAA in response to averted gaze faces - the opposite pattern of that elicited in neurotypical children (Lauttia et al., 2019). Findings in adults using fMRI and EEG source localization have linked asymmetry of the dorsolateral prefrontal cortex (dlPFC) to both approach/avoidance motivation and FAA (Berkman and Lieberman, 2010; Smith et al., 2018; Spielberg et al., 2011). In the current study, we therefore used functional nearinfrared spectroscopy (fNIRS) to examine frontal asymmetry within infants' bilateral dlPFC.

Oxytocin is a neurohormone which has been linked to several functions critical for social cognition. In adults, intranasal oxytocin administration has been linked to enhanced emotion recognition (Leknes et al., 2013; Lischke et al., 2012; Shahrestani et al., 2013) and a concomitant increase in the recruitment of attentional resources when viewing faces (Prehn et al., 2013). Furthermore, mounting evidence suggests that variability in endogenous or exogenous oxytocin levels impacts emotion processing in a valence-dependent manner (Kemp and Guastella, 2011). This line of research suggests that heightened oxytocin exposure is associated with increased brain or behavioral responding to positive stimuli and a simultaneous attenuated response to negative stimuli in adults (Gamer et al., 2010; Kirsch et al., 2005; Krol et al., 2014b; Marsh et al., 2010), non-human primates (Parr et al., 2013), and human infants (Krol et al., 2015a; Krol et al., 2019b; Krol et al., 2015b). Variability in oxytocin function may therefore play a critical role in infants' perception and interpretation of social cues, which may in turn impact motivation to approach or withdraw from early interaction partners. One such variation in the endogenous oxytocin system is in the ectoenzyme CD38 (Cluster of Differentiation 38). CD38 is critical 
to the function of endogenous oxytocin due to its regulation of calcium signaling during oxytocin release (Bartz and McInnes, 2007; Jin et al., 2007; Tolomeo et al., 2020). In mice, knockout models that lack the Cd38 gene have been used as a rodent model for ASD (Higashida et al., 2011). These knockout mice display extensive socio-behavioral deficits such as amnesia for conspecifics and impaired maternal caregiving behavior (Jin et al., 2007). In humans, naturally occurring genetic variation of the $C D 38$ gene in the form of the single-nucleotide polymorphism (SNP) rs3796863 has been associated with ASD diagnosis. Specifically, the C allele is considered a "risk" allele, as it is overrepresented in ASD as compared to neurotypical populations (Lerer et al., 2010; Munesue et al., 2010). Findings for MDD and $\mathrm{SAD}$ are more mixed with some studies reporting increased risk associated with the CC genotype (Tabak et al., 2016), whereas other studies report an increased risk associated with the AA genotype (McQuaid et al., 2016). In non-clinical samples, $C D 38$ has been associated with baseline oxytocin levels as well as neural reactions that indicate less engagement and more avoidance of social stimuli. Specifically, neurotypical adults with the CC ASD risk genotype (two copies of the "risky" C allele), as compared to non-risk A-allele carriers, have reduced plasma oxytocin (Feldman et al., 2012) and exhibit less efficient processing of social stimuli as evidenced by slower reaction times and increased fusiform activation to affective faces and social scenes (Sauer et al., 2012). In 7-month-old infants, this CD38 variation has been linked to individual differences in attention to emotional eye cues (Krol et al., 2015a), suggesting that CD38 plays a role in accounting for differences in infant social behavior. Despite links of both frontal asymmetry and $C D 38$ genotype to psychopathology, the existing literature does not contain any studies linking frontal asymmetry and $C D 38$ genotype, nor any examples linking $C D 38$ to other behavioral measures of social motivation. Most importantly for the context of the current study, no prior study has examined $C D 38$ genotype and risk for psychopathology as indexed by social behavior and FAA during infancy.

The current study combines genetics, neuroimaging and eyetracking to examine whether and how genetic variability within the oxytocinergic system relates to infant social information processing and motivation. Specifically, we measured dlPFC frontal asymmetry - known as a critical 
neuroendophenotype for disorders characterized by social withdrawal - to investigate variability in the motivation to approach or withdraw from smiling and frowning individuals. Following the frontal asymmetry measurements, we assessed infants' looking preferences for those individuals now displaying a neutral expression using eyetracking. We hypothesized that infants with the non-risk CA/AA genotypes would show heightened approach to smiling individuals, indexed by greater left dlPFC asymmetry and person preference for the smiling individual, and heightened withdrawal from frowning individuals, indexed by greater right dlPFC asymmetry and reduced or absent person preference for the frowning individual. We hypothesized that infants with the CC ASD risk genotype may show atypical social information processing and motivational tendencies in the form of increased right dlPFC asymmetry and decreased person preference when viewing smiling identities, compared to infants in the CA/AA genotype group.

\section{Material and methods}

\subsection{Participants}

Ninety-seven 11-month-old infants of Western European descent (48 females) participated in this study. All infants resided in Leipzig, a metropolitan German city of about 600,000 people. Based on an exclusion criterion established prior to data collection, 20 infants were excluded because they did not provide at least two artifact-free trials per condition in the fNIRS paradigm. Thus, the final sample comprises 77 infants (36 females) $\left(M_{\text {age }}=339.84\right.$ days, $\left.S D=6.77\right)$ born at standard birth weight $(>2,500$ grams) and gestational age (>38 weeks). Note that minimum sample size was determined based on a literature review of comparable neuroimaging by CD38 genotype designs (Sauer et al., 2012; Tabak et al., 2020), but chosen to be larger to account for standard attrition rates in infant neuroimaging studies. Eighteen infants were delivered by caesarean section, while 59 underwent standard vaginal births. No medical issues regarding development were reported at the time of testing, and there was no reported history of autism spectrum disorder (ASD) in any of the parents or older siblings of the infants. All but 11 infants had mothers on parental leave at the time of testing. Parity ranged from 0 to 2 older siblings $(M=$ 
$0.58, S D=0.70)$, and mothers reported an average education of 17 years $(S D=3.71)$. Parents provided written informed consent and were compensated with travel money, a toy for the infant, and a printed photograph of their infant in the fNIRS cap. All procedures were approved by the Leipzig University Medical School Ethics Committee and were conducted in accordance with the Declaration of Helsinki.

\subsection{Procedure}

2.2.1. Stimuli. The experimental design consisted of an fNIRS paradigm (impression formation phase) immediately followed by an eyetracking paradigm (person preference test), introduced and described in detail in Krol \& Grossmann (2020). During the impression formation phase, infants viewed four female identities while fNIRS was recorded; each identity consistently displayed the same expression-gaze combination: a) smile with direct gaze, b) smile with averted gaze, c) frown with direct gaze, or d) frown with averted gaze. This was immediately followed by a person preference test using eyetracking. Here, infants were shown side-by-side presentations of the four identities, now displaying neutral expressions.

2.2.2. Impression formation phase: fNIRS. Infants were seated on a parent's lap in a quiet, dimly lit room, facing a $52 \times 32 \mathrm{~cm}$ monitor at a distance of approximately $60 \mathrm{~cm}$. A camera attached to the bottom of the presentation screen recorded infant behavior for online and offline tracking of attention to each trial. The fNIRS paradigm was designed and presented in Presentation software (Neurobehavioral Systems, MA). Infants viewed 14 blocks of four randomized trials, each trial comprising a baseline (vegetable) stimulus followed by one of the four identities presenting an expression-gaze combination. Each block was comprised of four trials, containing one example each of an identity smiling with direct gaze, an identity smiling with averted gaze, an identity frowning with direct gaze, and an identity frowning with averted gaze (Figure 1A). Every block began with an attention-getter to keep infants alert and to orient them to the center of the screen (a shaking rattle, as described in Krol et al., 2015a). Facial stimuli were presented pseudo-dynamically through presentation of three photographs of the same identity: 1) a neutral expression with the non-target gaze (250 ms), 2) a neutral expression with the target gaze (250 ms), and finally, 3) the target expression combined with the target gaze (700 ms). This 
sequence repeated five times to create the illusion of an individual shifting and maintaining their gaze (direct or averted) while subsequently shifting from a neutral expression to either a smile or frown. Note that identity-expression-gaze combinations were counterbalanced across infants to control for potential identity effects. Infants were shown an average of 25.65 total trials (range $=10$ to $46 ; S D=7.57$ ).

fNIRS data were recorded using a NIRScout system and NIRStar acquisition software (NIRx, Berlin, Germany), using the same parameters and calibration as in our previous work (Krol and Grossmann, 2020; Krol et al., 2019b). Hemoglobin absorption was measured with 32 optodes (16 sources, 16 detectors) placed at approximately $2 \mathrm{~cm}$ distance over frontal and temporal cortices on a custom-built elastic cap (EasyCap, Germany). This arrangement comprised 49 channels (source-detector pairs) from which to measure localized hemodynamic activity. Near-infrared light was emitted at two wavelengths $(760 \mathrm{~nm}$ and $850 \mathrm{~nm})$ with a power of $5 \mathrm{~nm} /$ wavelength. Data were recorded at a sampling rate of 6.25 Hz.

\subsubsection{Person preference test: Eyetracking. Upon completion of the fNIRS paradigm, caps were} removed and infants underwent the eyetracking paradigm using Tobii Studio 3.2 (Danderyd, Sweden). Five-point calibration was conducted prior to collection using the same methods as reported in our previous work (Krol and Grossmann, 2020; Krol et al., 2015a). Fifteen-second trials were constructed as side-by-side pairings of two identities at a time (Figure 1B). In contrast to the fNIRS paradigm, identities were now presented with neutral, direct-gaze expressions and were paired to assess person preference for expression (i.e., the two actresses who maintained direct gaze (smile vs. frown)) and person preference for gaze direction (i.e., the two actresses who smiled (direct vs. averted gaze)). Four total comparisons were possible, and each was presented twice for a total of eight trials. Each trial was preceded by the same audiovisual attention-getter as in the fNIRS paradigm.

\subsection{Genotyping}

Saliva was collected using assisted-collection sponges and kits from DNA Genotek (Ottawa, Canada; CS-2 sponges and OG-250 kits). Samples were stored at room temperature until DNA isolation. DNA was isolated using the manual purification protocol from DNA Genotek. Genotyping of CD38 
rs3796863 was performed with a 5'-nuclease assay using primers and probes from Applied Biosystems (TaqMan® SNP Genotyping Assay). PCR was conducted with HotStarTaq Plus DNA polymerase and Qsolution (Qiagen, Venlo, Netherlands) in a Biorad C1000 thermocycler with a CFX96 fluorescence reading module, using the following thermal protocol: Denaturation at $95{ }^{\circ} \mathrm{C}$ for five minutes; followed by cycling: $95{ }^{\circ} \mathrm{C}$ for $15 \mathrm{~s}, 60^{\circ} \mathrm{C}$ for one minute; for 45 cycles.

\begin{tabular}{|l|l|l|l|}
\hline & CC & CA & AA \\
\hline Females & 12 & 20 & 4 \\
& $33.3 \%$ & $55.6 \%$ & $11.1 \%$ \\
\hline Males & 22 & 18 & 1 \\
& $53.7 \%$ & $43.9 \%$ & $2.4 \%$ \\
\hline Total & 34 & 38 & 5 \\
& $44.2 \%$ & $49.4 \%$ & $6.5 \%$ \\
\hline
\end{tabular}

Table 1. CD38 rs3796863 genotype frequencies separated by gender. Note there is no significant impact of gender on genotype frequency, $\chi^{2}(2)=4.54, p=0.103$.

\subsection{Questionnaires}

Demographic information from the mother (parity, education, age, method of delivery, breastfeeding behavior, subjective stress level) was assessed using an in-house questionnaire (Krol et al., 2014a; Krol et al., 2015a). In order to identify and control for potential confounds with infant CD38 genotype, we conducted group comparisons for all demographic data, including the above-mentioned maternal factors as well as infant age and gender. None of these factors associated with CD38 genotype; all $p$-values $>0.05$ (see Table 1 for $C D 38$ genotype frequencies separated by gender, and Table S1 to view the descriptive statistics and null associations of infant $C D 38$ genotype on reported demographic variables).

\subsection{Data analysis}

2.5.1. fNIRS analysis. Videos from each session were manually coded for infant looking duration to each trial. Trials were only included if infants attended to the screen at least four of the six seconds for 
which both baseline and face stimuli were presented and were visually inspected for motion artifacts. Trials with motion artifacts were removed from further analyses. Using the Matlab-based software Nilab2 (NIRx, Germany), data were filtered with a $0.2-\mathrm{Hz}$ low-pass filter in order to remove fluctuations due to infant heart rate and a high-pass filter of $0.083 \mathrm{~Hz}$ (12 seconds) to remove changes too slow to be related to experimental stimuli (i.e., fluctuations due to drift). Measurements were converted into oxygenated hemoglobin (oxy- $\mathrm{Hb})$ and deoxygenated hemoglobin (deoxy-Hb) absorption using the modified BeerLambert law. Boxcar functions corresponding to the four stimulus conditions were convolved with a standard hemodynamic response function based on the stimulus length parameter (Boynton et al., 1996). The average concentration changes of oxy-Hb and deoxy- $\mathrm{Hb}$ in response to each stimulus condition were extracted for each channel, for each individual infant. Infants contributed an average of five attended, artifact-free fNIRS trials per condition (see 3. Results).

As stated above, the dlPFC has been posited as an underlying brain region for FAA scores derived from EEG (Smith et al., 2018). Regions of interest (ROIs) containing the bilateral dorsolateral prefrontal cortex (dlPFC) were created by referencing anatomical sources of the infant 10-20 system (Kabdebon et al., 2014) and mapping our source-detector template onto infant brain space in NIRSite (NIRx) (Fonov et al., 2011) (See Figure S1). Visualizations of cortical projections were created in nirsLAB software (NIRx), which projects fNIRS channels onto adult MNI brain space (locked to 10-20 coordinates) (Figure 2). Hemispheric asymmetry scores were created by subtracting right dlPFC oxy-Hb response from left dlPFC oxy-Hb response. Thus, scores above zero indicate increased brain response in the left hemisphere as compared to right (left asymmetry: approach), and scores below zero indicate increased brain response in the right hemisphere as compared to left (right asymmetry: withdrawal). Note that this is different from the standard right minus left calculation used for EEG-derived FAA, because alpha power has an inverse relationship with brain activation (Allen et al., 2004). This is not the case with oxy- $\mathrm{Hb}$, for which increases are thought to reflect enhanced brain activation through neurovascular coupling (Lloyd-Fox et al., 2010). Our subtraction of left minus right is in line with fMRI asymmetry 
studies, which also capture hemodynamic measurements (Herrington et al., 2010; Kaur et al., 2020; Morys et al., 2020; Zotev et al., 2016).

\subsubsection{Eyetracking analysis. As in our fNIRS analyses, eyetracking analysis has been thoroughly} described in (Krol and Grossmann, 2020). Areas of interest comprising the eye region of each facial stimulus were created within Tobii Studio. The total looking duration to the eye region of each individual was extracted per infant, per trial type. Looking bias scores were computed by calculating the duration of looking time to one individual divided by total looking time to both individuals. This yielded four bias scores for each infant: a) gaze bias for individuals who presented frowns: frowning direct vs. frowning averted, b) gaze bias for individuals who smiled: smiling direct vs. smiling averted, c) expression bias for individuals who presented direct-gaze expressions: smiling direct vs. frowning direct, and d) expression bias for individuals who presented averted-gaze expressions: smiling averted vs. frowning averted. Performance on the eyetracking task crucially depended on infants' attention during the fNIRS task. The average trials viewed per condition during fNIRS was included as a covariate in all eyetracking analyses to account for differing experience with, and memory of, the emotional presentations.

\section{Results}

\subsection{Frontal asymmetry patterns to direct-gaze emotional expressions differ by CD38 genotype.}

Frontal asymmetry scores were entered into a repeated-measures ANOVA containing expression (smile or frown) and gaze (direct or averted) as within-subject factors, and CD38 genotype (ASD risk $[\mathrm{CC}]$, no-risk [CA/AA]) as a between-subjects factor. A three-way interaction was revealed, $F(1,75)=$ 4.67, $p=0.034, \eta p^{2}=0.059$. To examine this interaction further, we employed repeated-measures ANOVAs for each gaze direction separately. Using this method, we found that the interaction between expression and $C D 38$ was only present for the direct gaze condition, $F(1,75)=6.204, p=0.015, \eta p^{2}=$ 0.076. There was no expression by $C D 38$ interaction within averted gaze stimuli $(F(1,75)=0.935, p=$ $0.337, \eta p^{2}=0.012$ ). Specifically, when viewing direct-gaze expressions, CA/AA non-risk infants showed the predicted response: greater left asymmetry (approach) to smiling, direct-gaze faces, and greater right asymmetry (withdrawal) to frowning, direct-gaze faces. In contrast to the CA/AA infants, CC ASD risk 
infants displayed increased right asymmetry to the smiling, direct-gaze individuals, indexing the motivation to withdraw from friendly-appearing individuals (Figure 3). We further elucidated this threeway interaction by assessing the impact of expression and gaze on frontal asymmetry for each genotype group separately. Here, we find an expression by gaze interaction only within the non-risk CA/AA carriers, $F(1,42)=5.162, p=0.028, \eta p^{2}=0.109$. These infants again display the predicted response pattern that was absent in CC risk infants, $F(1,33)=0.515, p=0.478, \eta p^{2}=0.015$ : greatest left asymmetry to direct-gaze, as compared to averted-gaze, smiling faces, and greatest right asymmetry to direct-gaze, as compared to averted-gaze, frowning faces (Figure 3). Taken together, these findings demonstrate that CC risk infants display atypical patterns of approach and avoidance tendencies as indexed by frontal brain asymmetry. Note that based on the existing literature reporting sex differences in symptomatology and diagnosis of ASD (Halladay et al., 2015), we repeated this analysis with sex of the infant included. This did not substantially impact the significance of our three-way interaction $(p=0.038)$ (see Supplementary Materials for exploratory analyses with infant sex).

To consider the specificity of our dlPFC-derived asymmetry findings, we created asymmetry scores from a parietal ROI to serve as a comparative control. In line with the methods of Davidson and Fox (1982), who compared frontal electrode-derived asymmetry to parietal electrode-derived asymmetry in 10-month-old infants, we created asymmetry scores for two channels in parietal cortex located at CP3 and CP4 of the 10-20 coordinate system (Figure S1). This analysis revealed no interaction between expression, gaze, and CD38 genotype on parietal asymmetry $(F(1,75)=0.037, p=0.847)$, which indicates that our findings are restricted to the frontal cortex. This is thus similar to what has been reported in prior work using FAA.

\subsection{Person preference for smiling and frowning individuals differs by CD38 genotype.}

Univariate ANOVAs were performed on looking bias scores, with $C D 38$ genotype as a fixed factor and average trials viewed during the fNIRS session as a covariate. We found that when infants were shown neutral expressions of the two individuals who previously presented direct-gaze expressions (smiling direct vs. frowning direct), looking bias differed by $C D 38$ genotype, $F(1,72)=4.705, p=0.033$. 
Specifically, infants in the non-risk genotype group (CA/AA) displayed a looking preference for the individual who had previously smiled at them, while infants in the ASD risk genotype group (CC) displayed a looking preference for the individual who previously frowned (Figure 4). We found no impact of $C D 38$ genotype on the looking bias to individuals presenting expressions with an averted gaze, nor for either of the direct vs. averted gaze comparisons (all $p$-values $>0.05$ ).

\section{Discussion}

Our findings demonstrate that genetic variability in the oxytocin system impacts infants' motivational tendencies for social approach and withdrawal. Indexed by fNIRS-derived frontal asymmetry, infants in the ASD non-risk genotype group exhibited the predicted patterns of social approach and withdrawal. Specifically, they displayed approach towards smiling individuals reflected in increased left frontal asymmetry and withdrawal from frowning individuals reflected in increased right frontal asymmetry. In contrast, infants in the ASD risk genotype group - linked to reduced oxytocin levels in previous work - exhibited frontal asymmetry responses indexing withdrawal from smiling, direct-gaze faces and no aversion of frowning faces. Our results further show that $C D 38$ genotype was associated with differences in infants' behavioral preference for frowning or smiling individuals, measured through eyetracking. Infants in the ASD non-risk genotype group displayed a looking preference for the person who had previously smiled at them, whereas infants in the ASD risk genotype group displayed a looking preference for the person who had frowned at them. This shows that genetic variation is linked to variability in social motivational processes reflected in infants' brain and behavioral responses. This supports the notion that variability in the oxytocinergic system contributes to individual differences in brain systems involved in social motivational processes from early in human development with implications for atypical social development (Chevallier et al., 2012).

Our primary analyses demonstrate the impact of $C D 38$ genotype on neural and behavioral indices of approach and withdrawal when viewing individuals with direct gaze. This is in line with a host of studies showing that infants from early in ontogeny are sensitive to eye contact and that direct gaze serves as an important communicative cue signaling that the information conveyed through the face is self- 
relevant to the infant (Csibra and Gergely, 2009; Farroni et al., 2002; Grossmann, 2015). Notably, when analyzing our frontal asymmetry data separately within $C D 38$ genotype groups, we obtained the predicted and presumably typical pattern of motivational brain responses reflected in an interaction effect of emotional expression and gaze direction only within CA/AA non-risk infants. Specifically, our results indicate that infants in the ASD non-risk genotype group displayed increased approach indexed by left frontal asymmetry to smiling, direct-gaze faces and increased withdrawal indexed by right frontal asymmetry to frowning, direct-gaze faces. Taken together, our fNIRS results demonstrate that infants in the ASD risk group show frontal asymmetry responses that do not differentiate between emotions and gaze directions, suggesting atypical social-motivational information processing.

The current findings increase our understanding of the role of biological diathesis in the development of ASD. While Gabard-Durnam and colleagues (2015) showed a link between general genetic risk for ASD and FAA at rest, the current study provides evidence of a clear relation between a specific genetic variant and neural response and behavior, both of which are context-dependent. This is advantageous in considering the etiology of ASD and for the development of early, non-invasive interventions. By identifying $C D 38$ genotype as a specific marker associated with frontal asymmetry, the current study provides a clear target for early intervention - the oxytocinergic system. The study also identifies a specific context in which to intervene, during the presence of positive social interaction partners. Such interventions seem viable, considering that: 1) a large body of research has linked parent behaviors to increased infant oxytocin levels (see Scatliffe, Casavant, Vittner, \& Cong, 2019 for a systematic review) and 2) recent work indicating epigenetic upregulation of endogenous oxytocin receptor with increased maternal engagement (Krol et al., 2019a). Early interventions focused on facilitating oxytocin function - through its impact on social brain and behavioral motivation - could help to remediate social symptom severity among at-risk infants who are later diagnosed with ASD. Furthermore, if interventions do resolve abnormal frontal asymmetry, they may act on a risk factor for overall autism symptom severity (Schiltz et al., 2018), which systematic reviews have indicated are more resistant to gold-standard treatments later in development than specific clusters of social deficits (see 
Tachibana et al., 2017; Yu et al., 2020 for meta-analyses of applied behavior analysis based interventions).

The findings presented also provide insight into the biological bases of the development of childhood temperament and emotions. An approach/avoidance framework of individual differences in early infant emotional responses has been previously described as "the raw material on which experience and environmental input operate to shape behavioral or later personality styles" (Sullivan, 2014). One domain in which individual differences in early approach/avoidance tendencies might manifest is social responsiveness. The current findings point to a potential pathway by which genetic differences may manifest as differences in social responsiveness. Infants at genetic risk for being less responsive to oxytocin may attend and react differently to emotional faces than healthy infants, which in turn might manifest as later differences in social awareness, social cognition, social communication, social motivation and ultimately overt behavior (Grossmann et al., 2018). These social responsiveness factors have been previously linked to childhood temperament factors of surgency/extraversion, negative affect, and effortful control (see Salley et al., 2013 for a more detailed examination of the relationship between these variables at two to four years of age).

A final point warranting discussion is that of the exact neurobiological mechanisms underlying the observed effects on social and motivational processes in infancy. While $C D 38$ genotype is associated with baseline oxytocin level in adults (Feldman et al., 2012), we did not assess peripheral oxytocin within our infant cohort. Future work delineating the presumed interaction between endogenous oxytocin and CD38 genotype is warranted, as it remains an open question as to how variability at this particular SNP exerts its reported effects on social motivation. Exploring salivary or urine oxytocin level at rest and during perception of social cues in conjunction with $C D 38$ genotype in future studies might help provide insight into the molecular mechanisms underpinning variability in social motivation among infants. In addition, while CD38 has been specifically linked to oxytocin release, it is likely that the downstream socio-behavioral effects of this SNP are due to an intricate interaction of several molecular systems. For example, the oxytocinergic system has strong links with other neuromodulatory systems, including 
dopamine and serotonin, which play critical roles in reward processing and mood (Baskerville and Douglas, 2010). In particular, there is evidence that oxytocin modulates social reward and motivation through excitation of dopaminergic neurons in the ventral tegmental area (Hung et al., 2017), as well as through a coordinated effort with serotonin within the nucleus accumbens (Dolen et al., 2013).

The current study's demonstration of variability of social approach and withdrawal tendencies as a function of genetic variation in the oxytocin system (CD38) critically advances emerging work indicating that the oxytocin system plays an important role in social brain and behavioral development during infancy (Krol et al., 2015a; Krol et al., 2019b). Specifically, these findings are in line with existing accounts emphasizing the role of oxytocin in enhancing the salience of affiliative social signals while simultaneously reducing responding to threatening social signals (Kemp and Guastella, 2011). Here, we add an important developmental dimension to the emerging picture regarding links between the oxytocinergic system and variability in social-motivational brain processes with potential clinical relevance for the early identification of neurodevelopmental disorders such as ASD. Overall, this study advances our understanding of the role of the oxytocin system in social cognition and behavior, pointing to individual differences in a motivational brain mechanism linked to prefrontal cortex function and variability in social motivation. 


\section{Acknowledgements}

We are grateful to all families who participated in this study. We thank Caterina Boettcher, Jenny Tippmann, and Katharina Kerber for their assistance with infant testing and data preparation at the Max Planck Institute for Human Cognitive and Brain Sciences. This work was funded by the Max Planck Society, the National Science Foundation \#2017229 (TG), an International Max Planck Research School (IMPRS NeuroCom) scholarship (KK), an NIH NRSA T32 Research Training in Neuroendocrinology Fellowship (KK), and a Hartwell Biomedical Research Fellowship (KK). This work was presented in both oral and poster format at the $74^{\text {th }}$ Annual Meeting of the Society of Biological Psychiatry; the abstracts of which have been published in Biological Psychiatry. A preprint of this manuscript was uploaded on PsyArXiv.

\section{Disclosures}

The authors (KK, NN, MM, PL, RE, TG) have nothing to disclose. 


\section{References}

Allen, J.J.B., Coan, J.A., Nazarian, M., 2004. Issues and assumptions on the road from raw signals to metrics of frontal EEG asymmetry in emotion. Biol Psychol 67, 183-218.

Bartz, J.A., McInnes, L.A., 2007. CD38 regulates oxytocin secretion and complex social behavior. BioEssays 29, 837-841.

Baskerville, T.A., Douglas, A.J., 2010. Dopamine and Oxytocin Interactions Underlying Behaviors: Potential Contributions to Behavioral Disorders. Cns Neurosci Ther 16, e92-e123. Berkman, E.T., Lieberman, M.D., 2010. Approaching the bad and avoiding the good: lateral prefrontal cortical asymmetry distinguishes between action and valence. J Cogn Neurosci 22, 1970-1979.

Boynton, G.M., Engel, S.A., Glover, G.H., Heeger, D.J., 1996. Linear systems analysis of functional magnetic resonance imaging in human V1. Journal of Neuroscience 16, 4207-4221. Chevallier, C., Kohls, G., Troiani, V., Brodkin, E.S., Schultz, R.T., 2012. The social motivation theory of autism. Trends Cogn Sci 16, 231-239.

Coan, J.A., Allen, J.J., Harmon-Jones, E., 2001. Voluntary facial expression and hemispheric asymmetry over the frontal cortex. Psychophysiology 38, 912-925.

Coan, J.A., Allen, J.J.B., 2003. Varieties of emotional experience during voluntary emotional facial expressions. Emotions inside Out 1000, 375-379.

Cowell, J.M., Decety, J., 2015. Precursors to morality in development as a complex interplay between neural, socioenvironmental, and behavioral facets. Proc Natl Acad Sci USA 112, 1265712662.

Csibra, G., Gergely, G., 2009. Natural pedagogy. Trends Cogn Sci 13, 148-153.

Davidson, R.J., Fox, N.A., 1982. Asymmetrical Brain Activity Discriminates between Positive and Negative Affective Stimuli in Human Infants. Science 218, 1235-1237.

Davidson, R.J., Marshall, J.R., Tomarken, A.J., Henriques, J.B., 2000. While a phobic waits: Regional brain electrical and autonomic activity in social phobics during anticipation of public speaking. Biol Psychiatry 47, 85-95.

Dolen, G., Darvishzadeh, A., Huang, K.W., Malenka, R.C., 2013. Social reward requires coordinated activity of nucleus accumbens oxytocin and serotonin. Nature 501, 179-+.

Farroni, T., Csibra, G., Simion, G., Johnson, M.H., 2002. Eye contact detection in humans from birth. Proc Natl Acad Sci USA 99, 9602-9605.

Feldman, R., Zagoory-Sharon, O., Weisman, O., Schneiderman, I., Gordon, I., Maoz, R., Shalev, I., Ebstein, R.P., 2012. Sensitive parenting is associated with plasma oxytocin and polymorphisms in the OXTR and CD38 genes. Biol Psychiatry 72, 175-181.

Fonov, V., Evans, A.C., Botteron, K., Almli, C.R., McKinstry, R.C., Collins, D.L., Grp, B.D.C., 2011. Unbiased average age-appropriate atlases for pediatric studies. NeuroImage 54, 313-327. Gamer, M., Zurowski, B., Buchel, C., 2010. Different amygdala subregions mediate valencerelated and attentional effects of oxytocin in humans. Proc Natl Acad Sci USA 107, 9400-9405. Grossmann, T., 2013. The early development of processing emotions in face and voice, in: Belin, P., Campanella, S., Ethofer, T. (Eds.), Integrating Face and Voice in Person Perception. Springer, New York, pp. 95-116.

Grossmann, T., 2015. The development of social brain functions in infancy. Psychol Bull 141, 1266-1287.

Grossmann, T., 2017. The Eyes as Windows Into Other Minds: An Integrative Perspective. Perspect Psychol Sci 12, 107-121. 
Grossmann, T., Missana, M., Krol, K.M., 2018. The neurodevelopmental precursors of altruistic behavior in infancy. Plos Biol 16, e2005281.

Grunewald, B.D., Greimel, E., Trinkl, M., Bartling, J., Grossheinrich, N., Schulte-Korne, G., 2018. Resting frontal EEG asymmetry patterns in adolescents with and without major depression. Biol Psychol 132, 212-216.

Halladay, A.K., Bishop, S., Constantino, J.N., Daniels, A.M., Koenig, K., Palmer, K., Messinger, D., Pelphrey, K., Sanders, S.J., Singer, A.T., Taylor, J.L., Szatmari, P., 2015. Sex and gender differences in autism spectrum disorder: summarizing evidence gaps and identifying emerging areas of priority. Mol Autism 6.

Hamlin, J.K., Wynn, K., Bloom, P., 2007. Social evaluation by preverbal infants. Nature 450, 557-U513.

Harmon-Jones, E., Gable, P.A., 2018. On the role of asymmetric frontal cortical activity in approach and withdrawal motivation: An updated review of the evidence. Psychophysiology 55. Herrington, J.D., Heller, W., Mohanty, A., Engels, A.S., Banich, M.T., Webb, A.G., Miller, G.A., 2010. Localization of asymmetric brain function in emotion and depression.

Psychophysiology 47, 442-454.

Higashida, H., Yokoyama, S., Munesue, T., Kikuchi, M., Minabe, Y., Lopatina, O., 2011. CD38 gene knockout juvenile mice: a model of oxytocin signal defects in autism. Biol Pharm Bull 34, 1369-1372.

Hung, L.W., Neuner, S., Polepalli, J.S., Beier, K.T., Wright, M., Walsh, J.J., Lewis, E.M., Luo, L.Q., Deisseroth, K., Dolen, G., Malenka, R.C., 2017. Gating of social reward by oxytocin in the ventral tegmental area. Science 357, 1406-1411.

Jin, D., Liu, H.X., Hirai, H., Torashima, T., Nagai, T., Lopatina, O., Shnayder, N.A., Yamada, K., Noda, M., Seike, T., Fujita, K., Takasawa, S., Yokoyama, S., Koizumi, K., Shiraishi, Y., Tanaka, S., Hashii, M., Yoshihara, T., Higashida, K., Islam, M.S., Yamada, N., Hayashi, K., Noguchi, N., Kato, I., Okamoto, H., Matsushima, A., Salmina, A., Munesue, T., Shimizu, N., Mochida, S., Asano, M., Higashida, H., 2007. CD38 is critical for social behaviour by regulating oxytocin secretion. Nature 446, 41-45.

Kabdebon, C., Leroy, F., Simmonet, H., Perrot, M., Dubois, J., Dehaene-Lambertz, G., 2014. Anatomical correlations of the international 10-20 sensor placement system in infants.

NeuroImage 99, 342-356.

Kaur, A., Chinnadurai, V., Chaujar, R., 2020. Microstates-based resting frontal alpha asymmetry approach for understanding affect and approach/withdrawal behavior. Sci Rep 10.

Kemp, A.H., Guastella, A.J., 2011. The role of oxytocin in human affect: A novel hypothesis. Curr Dir Psychol Sci 20, 222-231.

Kirsch, P., Esslinger, C., Chen, Q., Mier, D., Lis, S., Siddhanti, S., Gruppe, H., Mattay, V.S., Gallhofer, B., Meyer-Lindenberg, A., 2005. Oxytocin modulates neural circuitry for social cognition and fear in humans. J Neurosci 25, 11489-11493.

Krol, K.M., Grossmann, T., 2020. Impression Formation in the Human Infant Brain. Cereb Cortex Commun 1, tgaa070.

Krol, K.M., Kamboj, S.K., Curran, H.V., Grossmann, T., 2014a. Breastfeeding experience differentially impacts recognition of happiness and anger in mothers. Sci Rep 4, 7006.

Krol, K.M., Monakhov, M., Lai, P.S., Ebstein, R.P., Grossmann, T., 2015a. Genetic variation in CD38 and breastfeeding experience interact to impact infants' attention to social eye cues. Proc Natl Acad Sci USA 112, E5434-E5442. 
Krol, K.M., Moulder, R.G., Lillard, T.S., Grossmann, T., Connelly, J.J., 2019a. Epigenetic dynamics in infancy and the impact of maternal engagement. Sci Adv 5, eaay0680.

Krol, K.M., Puglia, M.H., Morris, J.P., Connelly, J.J., Grossmann, T., 2019b. Epigenetic modification of the oxytocin receptor gene is associated with emotion processing in the infant brain. Dev Cogn Neurosci 37.

Krol, K.M., Rajhans, P., Missana, M., Grossmann, T., 2014b. Duration of exclusive breastfeeding is associated with differences in infants' brain responses to emotional body expressions. Front Behav Neurosci 8, 459.

Krol, K.M., Rajhans, P., Missana, M., Grossmann, T., 2015b. Duration of exclusive breastfeeding is associated with differences in infants' brain responses to emotional body expressions. Front Behav Neurosci 8, 459.

Lauttia, J., Helminen, T.M., Leppanen, J.M., Yrttiaho, S., Eriksson, K., Hietanen, J.K., Kylliainen, A., 2019. Atypical Pattern of Frontal EEG Asymmetry for Direct Gaze in Young Children with Autism Spectrum Disorder. J Autism Dev Dis 49, 3592-3601.

Leknes, S., Wessberg, J., Ellingsen, D.M., Chelnokova, O., Olausson, H., Laeng, B., 2013. Oxytocin enhances pupil dilation and sensitivity to 'hidden' emotional expressions. Soc Cogn Affect Neurosci 8, 741-749.

Lerer, E., Levi, S., Israel, S., Yaari, M., Nemanov, L., Mankuta, D., Nurit, Y., Ebstein, R.P., 2010. Low CD38 expression in lymphoblastoid cells and haplotypes are both associated with autism in a family-based study. Autism Res 3, 293-302.

Lischke, A., Berger, C., Prehn, K., Heinrichs, M., Herpertz, S.C., Domes, G., 2012. Intranasal oxytocin enhances emotion recognition from dynamic facial expressions and leaves eye-gaze unaffected. Psychoneuroendocrinology 37, 475-481.

Lloyd-Fox, S., Blasi, A., Elwell, C.E., 2010. Illuminating the developing brain: The past, present and future of functional near infrared spectroscopy. Neurosci Biobehav R 34, 269-284.

Marsh, A.A., Yu, H.H., Pine, D.S., Blair, R.J., 2010. Oxytocin improves specific recognition of positive facial expressions. Psychopharmacology (Berl) 209, 225-232.

McQuaid, R.J., McInnis, O.A., Matheson, K., Anisman, H., 2016. Oxytocin and Social

Sensitivity: Gene Polymorphisms in Relation to Depressive Symptoms and Suicidal Ideation.

Front Hum Neurosci 10.

Milne, L., Greenway, P., Guedeney, A., Larroque, B., 2009. Long term developmental impact of social withdrawal in infants. Infant Behav Dev 32, 159-166.

Morys, F., Janssen, L.K., Cesnaite, E., Beyer, F., Garcia-Garcia, I., Kube, J., Kumral, D., Liem, F., Mehl, N., Mahjoory, K., Schrimpf, A., Gaebler, M., Margulies, D., Villringer, A., Neumann, J., Nikulin, V.V., Horstmann, A., 2020. Hemispheric asymmetries in resting-state EEG and fMRI are related to approach and avoidance behaviour, but not to eating behaviour or BMI. Hum Brain Mapp 41, 1136-1152.

Munesue, T., Yokoyama, S., Nakamura, K., Anitha, A., Yamada, K., Hayashi, K., Asaka, T., Liu, H.X., Jin, D., Koizumi, K., Islam, M.S., Huang, J.J., Ma, W.J., Kim, U.H., Kim, S.J., Park, K., Kim, D., Kikuchi, M., Ono, Y., Nakatani, H., Suda, S., Miyachi, T., Hirai, H., Salmina, A., Pichugina, Y.A., Soumarokov, A.A., Takei, N., Mori, N., Tsujii, M., Sugiyama, T., Yagi, K., Yamagishi, M., Sasaki, T., Yamasue, H., Kato, N., Hashimoto, R., Taniike, M., Hayashi, Y., Hamada, J., Suzuki, S., Ooi, A., Noda, M., Kamiyama, Y., Kido, M.A., Lopatina, O., Hashii, M., Amina, S., Malavasi, F., Huang, E.J., Zhang, J., Shimizu, N., Yoshikawa, T., Matsushima, A., Minabe, Y., Higashida, H., 2010. Two genetic variants of CD38 in subjects with autism spectrum disorder and controls. Neurosci Res 67, 181-191. 
Parr, L.A., Modi, M., Siebert, E., Young, L.J., 2013. Intranasal oxytocin selectively attenuates rhesus monkeys' attention to negative facial expressions. Psychoneuroendocrinology 38, 17481756.

Prehn, K., Kazzer, P., Lischke, A., Heinrichs, M., Herpertz, S.C., Domes, G., 2013. Effects of intranasal oxytocin on pupil dilation indicate increased salience of socioaffective stimuli. Psychophysiology 50, 528-537.

Salley, B., Miller, A., Bell, M.A., 2013. Associations between Temperament and Social Responsiveness in Young Children. Infant Child Dev 22, 270-288.

Sauer, C., Montag, C., Wörner, C., Kirsch, P., Reuter, M., 2012. Effects of a common variant in the CD38 gene on social processing in an oxytocin challenge study: Possible links to autism. Neuropsychopharmacology 37, 1474-1482.

Schiltz, H.K., McVey, A.J., Barrington, A., Haendel, A.D., Dolan, B.K., Willar, K.S., Pleiss, S., Karst, J.S., Vogt, E., Murphy, C.C., Gonring, K., Van Hecke, A.V., 2018. Behavioral Inhibition and Activation as a Modifier Process in Autism Spectrum Disorder: Examination of SelfReported BIS/BAS and Alpha EEG Asymmetry. Autism Res 11, 1653-1666.

Shahrestani, S., Kemp, A.H., Guastella, A.J., 2013. The impact of a single administration of intranasal oxytocin on the recognition of basic emotions in humans: a meta-analysis.

Neuropsychopharmacology 38, 1929-1936.

Smith, E.E., Cavanagh, J.F., Allen, J.J.B., 2018. Intracranial source activity (eLORETA) related to scalp-level asymmetry scores and depression status. Psychophysiology 55.

Spielberg, J.M., Miller, G.A., Engels, A.S., Herrington, J.D., Sutton, B.P., Banich, M.T., Heller, W., 2011. Trait approach and avoidance motivation: lateralized neural activity associated with executive function. NeuroImage 54, 661-670.

Stewart, J.L., Bismark, A.W., Towers, D.N., Coan, J.A., Allen, J.J.B., 2010. Resting Frontal EEG Asymmetry as an Endophenotype for Depression Risk: Sex-Specific Patterns of Frontal Brain Asymmetry. Journal of Abnormal Psychology 119, 502-512.

Stewart, J.L., Coan, J.A., Towers, D.N., Allen, J.J., 2011. Frontal EEG asymmetry during emotional challenge differentiates individuals with and without lifetime major depressive disorder. J Affect Disord 129, 167-174.

Stewart, J.L., Coan, J.A., Towers, D.N., Allen, J.J., 2014. Resting and task-elicited prefrontal EEG alpha asymmetry in depression: support for the capability model. Psychophysiology 51, 446-455.

Sullivan, M.W., 2014. Infant expressions in an approach/withdrawal framework. J Genet Psychol 175, 472-493.

Tabak, B.A., Vrshek-Schallhorn, S., Zinbarg, R.E., Prenoveau, J.M., Mineka, S., Redei, E.E., Adam, E.K., Craske, M.G., 2016. Interaction of CD38 Variant and Chronic Interpersonal Stress Prospectively Predicts Social Anxiety and Depression Symptoms Over 6 Years. Clin Psychol Sci 4, 17-27.

Tabak, B.A., Young, K.S., Torre, J.B., Way, B.M., Burklund, L.J., Eisenberger, N.I., Lieberman, M.D., Craske, M.G., 2020. Preliminary Evidence That CD38 Moderates the Association of Neuroticism on Amygdala-Subgenual Cingulate Connectivity. Front Neurosci 14.

Tachibana, Y., Miyazaki, C., Ota, E., Mori, R., Hwang, Y., Kobayashi, E., Terasaka, A., Tang, J.L., Kamio, Y., 2017. A systematic review and meta-analysis of comprehensive interventions for pre-school children with autism spectrum disorder (ASD). PLoS One 12.

Thibodeau, R., Jorgensen, R.S., Kim, S., 2006. Depression, anxiety, and resting frontal EEG asymmetry: a meta-analytic review. J Abnorm Psychol 115, 715-729. 
Tolomeo, S., Chiao, B., Lei, Z., Chew, S.H., Ebstein, R.P., 2020. A Novel Role of CD38 and Oxytocin as Tandem Molecular Moderators of Human Social Behavior. Neurosci Biobehav R 115, 251-272.

Yu, Q., Li, E.Y., Li, L.G., Liang, W.Y., 2020. Efficacy of Interventions Based on Applied Behavior Analysis for Autism Spectrum Disorder: A Meta-Analysis. Psychiat Invest 17, 432443.

Zotev, V., Yuan, H., Misaki, M., Phillips, R., Young, K.D., Feldner, M.T., Bodurka, J., 2016. Correlation between amygdala BOLD activity and frontal EEG asymmetry during real-time fMRI neurofeedback training in patients with depression. Neuroimage-Clin 11, 224-238. 


\section{Figure Legend}

Figure 1. Stimulus presentation during fNIRS and eyetracking tasks. (A) Infants viewed four individuals displaying either a smile or frown with direct or averted gaze while fNIRS was recorded from bilateral dorsolateral prefrontal cortex (dlPFC). (B) Infants then underwent a person preference task using eyetracking. Specifically, infants viewed trials containing side-by-side presentation of the identities from the fNIRS task, now presenting a neutral expression with direct gaze. Note that due to copyright restrictions we have recreated the stimuli using photographs from the publicly available Karolinska Directed Emotional Faces (KDEF) database (www.kdef.se); $\mathrm{S}=$ smile, $\mathrm{F}=$ frown, $\mathrm{D}=\operatorname{direct}, \mathrm{A}=$ averted.

Figure 2. fNIRS cap template and ROIs. Shown is our fNIRS cap template mapped onto 10-20 space. International 10-20 coordinates are indicated by small gray dots, and relevant coordinates are labeled. Forty-nine channels (source-detector pairs) are presented as circles; those shaded in black are the channels used to create ROIs for bilateral dIPFC. Channels have been projected onto MNI brain space using NIRSite and nirsLAB software (NIRx) for readers' reference. Please see Figure S1 to view our full cap template in infant scalp and brain space.

Figure 3. Frontal asymmetry to direct-gaze emotional expressions differs by $C D 38$ genotype. Shown are the dlPFC-derived frontal asymmetry responses to smiling (green) and frowning (red) expressions stratified by CD38 genotype group (CC ASD risk genotype vs. non-risk A-allele carriers) and shown for direct and averted gaze separately. We find an interaction between $C D 38$ genotype and expression within the direct-gaze condition, $F(1,75)=6.204, p=0.015, \eta p^{2}=0.076$. Specifically, A-allele carriers demonstrate increased left asymmetry toward smiling faces, indexing approach, and increased right asymmetry toward frowning faces, indexing withdrawal. In contrast, infants with the CC ASD risk genotype display increased right asymmetry to smiling faces, indicating motivation to withdraw; ${ }^{*} p<$ $0.05,{ }^{\mathrm{t}} p<0.06$. 
Figure 4. Person preference for smiling and frowning individuals differs by $C D 38$ genotype. Infants viewed a side-by-side presentation of the two identities who previously smiled and frowned with direct gaze. Shown are the person preference scores for the two identities, as measured by eyetracking. Scores above $50 \%$ indicate a looking bias for the individual who smiled, scores below $50 \%$ indicate a looking bias for the individual who frowned. Person preference significantly differed by $C D 38$ genotype, $F(1,72)$ $=4.705, p=0.033$. Specifically, A-allele carriers showed a preference for the individual who smiled, while CC ASD risk infants showed a preference for the individual who frowned; ${ }^{*} p<0.05$. 
Figure 1.

A.

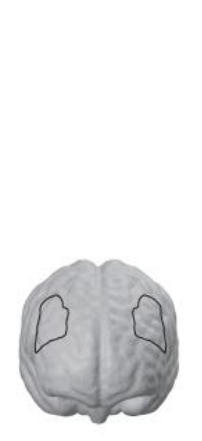

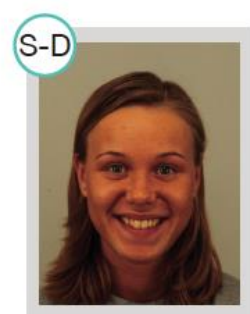

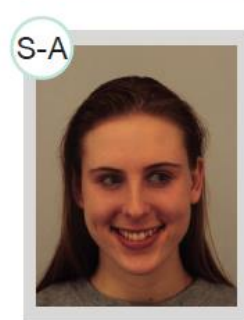

B.
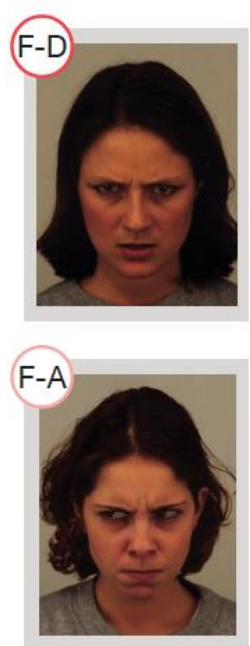

(S-D)

F-D

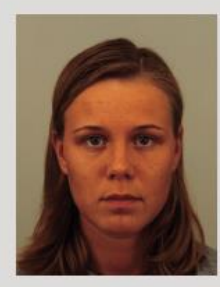

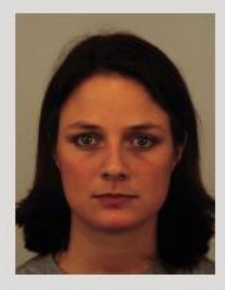


Figure 2.
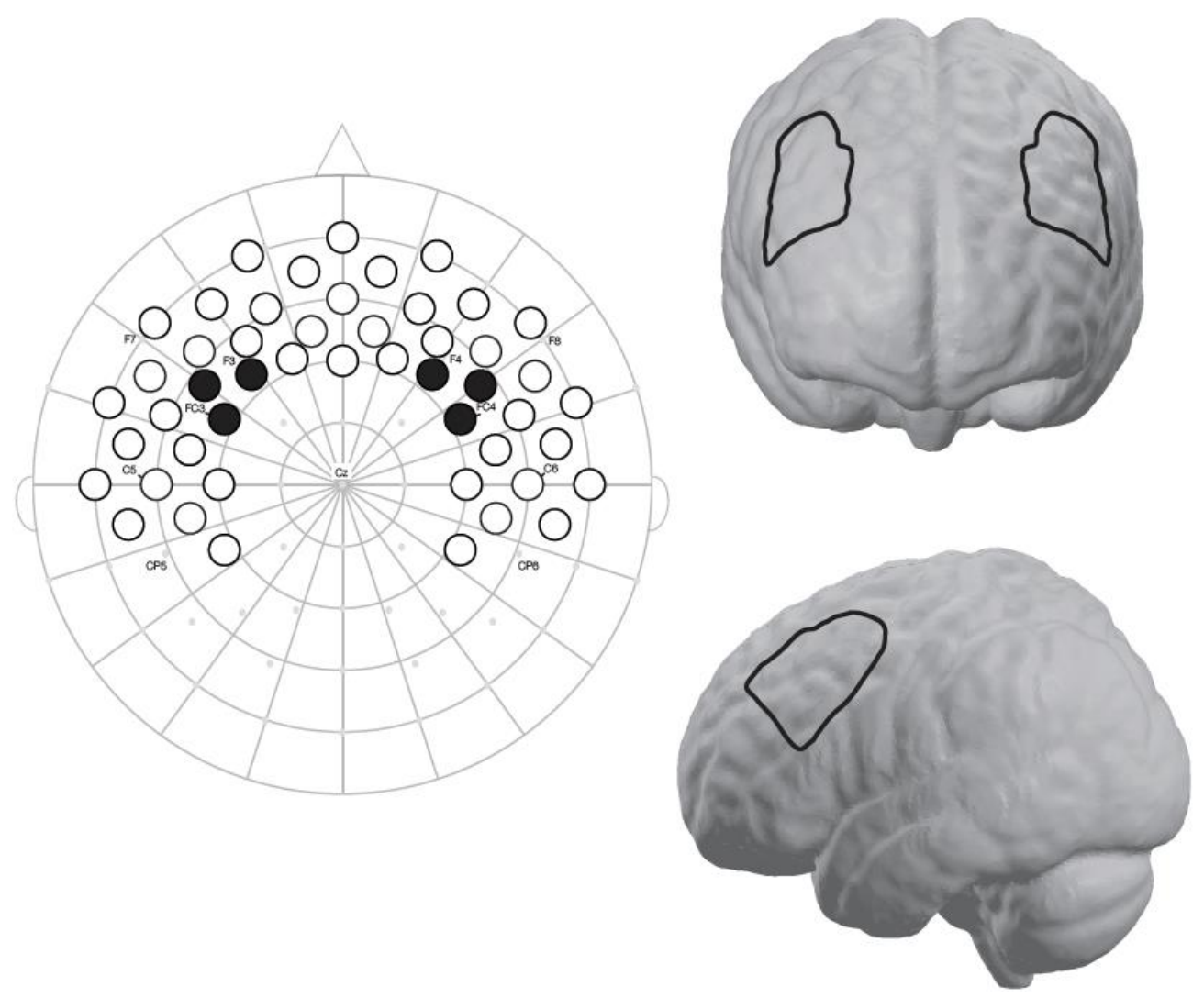
Figure 3.

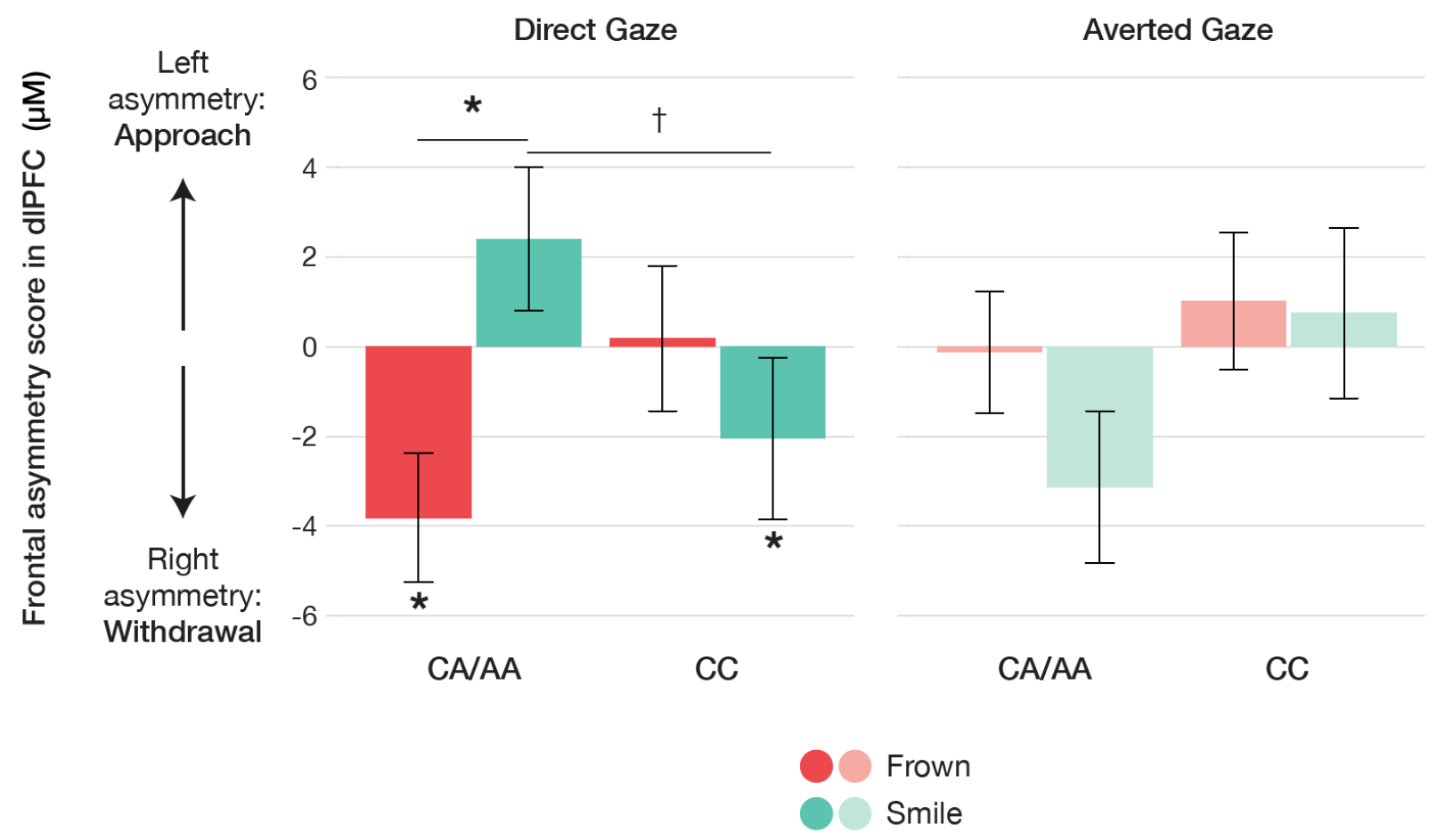


Figure 4.

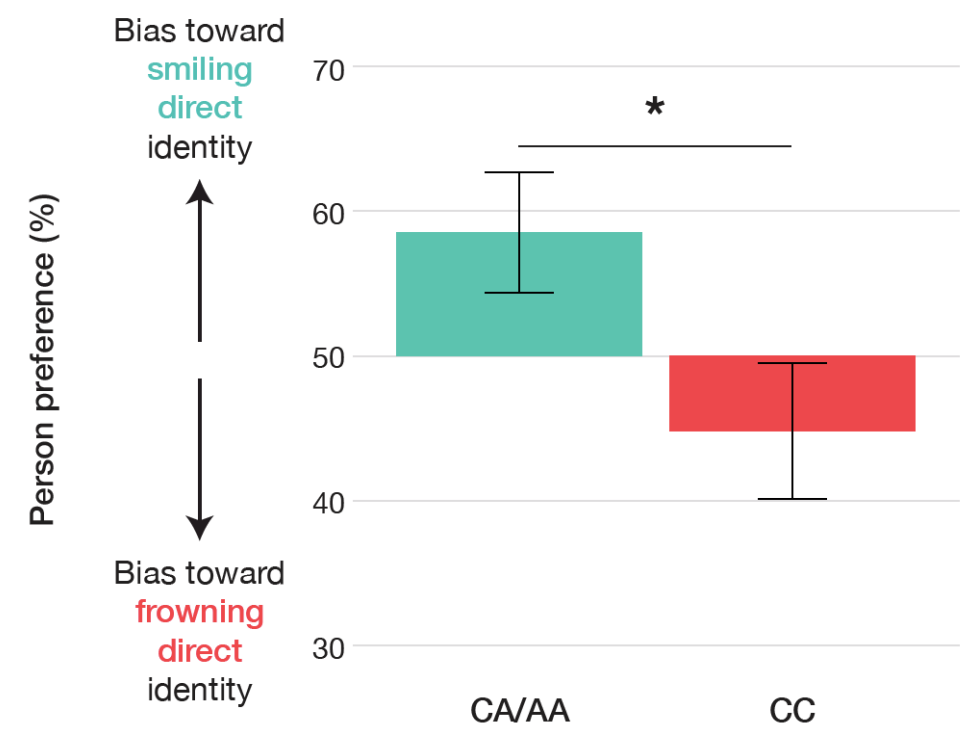




\section{Figure S1.}

A.

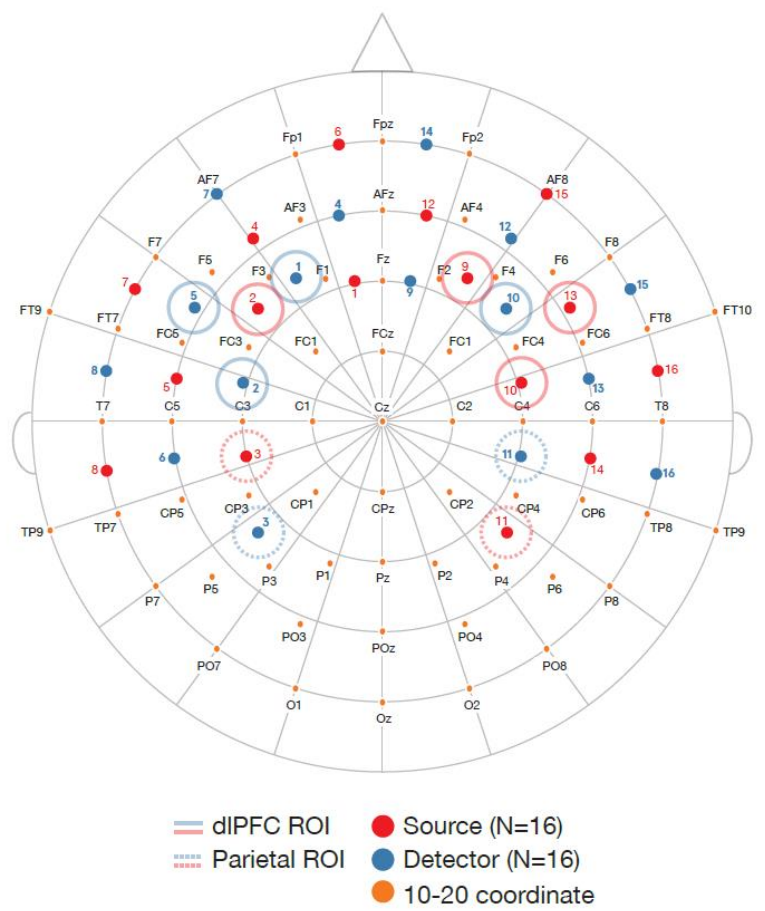

B.

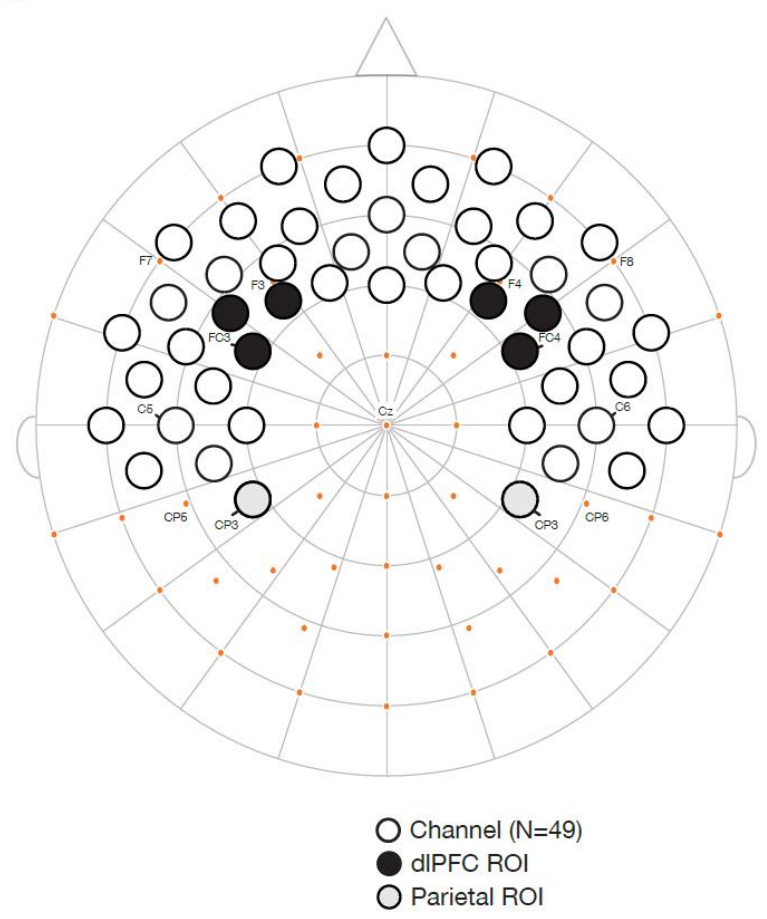

C.
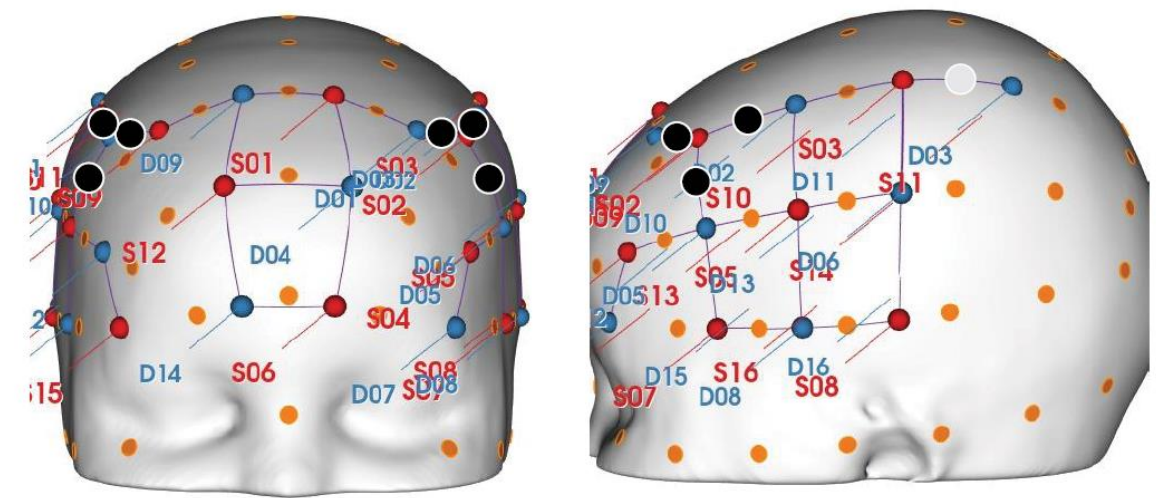

D.
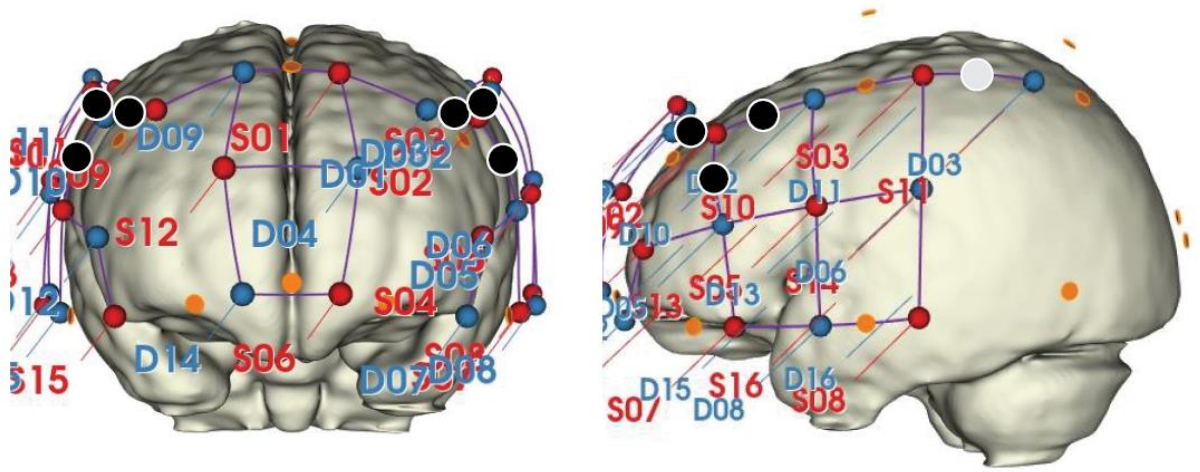